\title{
BMJ Global Health Shifting the dynamics: implementation of locally driven, mixed-methods modelling to inform schistosomiasis control and elimination activities
}

\author{
Cristin Alexis Fergus (D) ,, ${ }^{1,2}$ Bono Ozunga, ${ }^{3}$ Noah Okumu, ${ }^{4}$ Melissa Parker, ${ }^{5}$ \\ Solomon Kamurari, ${ }^{6}$ Tim Allen $^{1}$
}

To cite: Fergus CA, Ozunga B, Okumu N, et al. Shifting the dynamics: implementation of locally driven, mixedmethods modelling to inform schistosomiasis control and elimination activities. BMJ Global Health 2022;7:e007113. doi:10.1136/ bmjgh-2021-007113

Handling editor Seye Abimbola

- Additional supplemental material is published online only. To view, please visit the journal online (http://dx.doi.org/10. 1136/bmjgh-2021-007113)

Received 7 August 2021 Accepted 3 January 2022

Check for updates

C Author(s) (or their employer(s)) 2022. Re-use permitted under CC BY-NC. No commercial re-use. See rights and permissions. Published by BMJ.

For numbered affiliations see end of article.

Correspondence to Cristin Alexis Fergus; c.fergus@|se.ac.uk

\section{ABSTRACT}

Introduction The integration of more diverse perspectives into the development of evidence for decision-making has been elusive, despite years of rhetoric to the contrary. This has led to cycles of population-based health interventions which have not delivered the promised results. The WHO most recently set a target for schistosomiasis elimination by 2030 and called for cross-cutting approaches to be driven by endemic countries themselves. The extent to which elimination is feasible within the time frame has been a subject of debate.

Methods Systems maps were developed through participatory modelling activities with individuals working on schistosomiasis control and elimination activities from the village through national levels in Uganda. These maps were first synthesised, then used to frame the form and content of subsequent mathematical modelling activities, and finally explicitly informed model parameter specifications for simulations, using the opensource SCHISTOX model, driven by the participants.

Results Based on the outputs of the participatory modelling, the simulation activities centred around reductions in wate contact. The results of the simulations showed that mass drug administration, at either the current or target levels of coverage, combined with water contact reduction activities, achieved morbidity control in high prevalence Schistosoma mansoni settings, while both morbidity control and elimination were achieved in high prevalence $S$. haematobium settings within the 10-year time period.

Conclusion The combination of participatory systems mapping and individual-based modelling was a rich strategy which explicitly integrated the perspectives of national and subnational policymakers and practitioners into the development of evidence. This strategy can serve as a method by which individuals who have not been traditionally included in modelling activities, and do not hold positions or work in traditional centres of power, may be heard and truly integrated into the development of evidence for decision-making in global health.

\section{INTRODUCTION}

As described by the WHO's Director of the Department of Neglected Tropical Diseases (NTDs), the newest strategy to control and eliminate NTDs by 2030 (the 'NTD Road Map
Key questions

What is already known?

- The elimination of schistosomiasis as a public health programme has been shown to be feasible, as evidenced by previous case studies and predictive modelling estimates.

- However, the continued prioritisation of mass drug administration, with the minimal integration and lack of widespread financial support for alternative interventions, is not supported by this evidence.

- Further, there remains a disconnect between the rhetoric of country-driven, locally based solutions to global health problems, which have been shown to significantly improve impacts, and the reality of its widespread implementation

What are the new findings?

- In this study, we used participatory modelling to shape and inform mathematical modelling, demonstrating one of the possible strategies to integrate a wider range of perspectives in the form of individuals directly involved in the policy, oversight, and implementation of control and elimination activities within endemic countries.

- We conducted participatory systems mapping workshops with individuals at the village, district and nationa levels of the Uganda Ministry of Health, then used these outputs to select and inform the parameters of an opensource individual-based model.

- The results of this approach showed that achieving morbidity control and elimination was achievable within most recent time frame set forth by the WHO, once priority was given to complementary interventions.

What do the new findings imply?

- Incorporating the perspectives of individuals embedded in the biological and social systems of locations with endemic schistosomiasis has important, positive impacts on the development of evidence to support policy and practice.

- As has been suggested by previous evidence, and supported by this study, intervention strategies need to be tailored to local contexts, supported by a reorientation in the development of evidence for decision-making. 
to $\left.2030^{\prime}\right)^{1}$ was 'built on the principle of impact at country level through cross-cutting approaches, owned and driven by countries themselves, and augmented by coordinated support from partners'. ${ }^{2}$ Similar comments have been made specifically about the NTD, schistosomiasis, for which the primary interventions are mass deworming activities, referred to as preventative chemotherapy (PC) or mass drug administration (MDA), which are the distributions of deworming medicines to populations or subpopulations in defined geographical areas without individual diagnosis. Policymakers and practitioners have described the need to move from a top-down approach focused solely on MDA to integrated and adaptive strategies which are responsive to specific settings and populations. $^{3-6}$ These discussions are supported by empirical evidence in countries where schistosomiasis elimination has occurred, yet none of which achieved success through mass deworming strategies alone.

In addition to the lack of effectiveness in achieving elimination, meta-analyses have shown that mass deworming interventions targeting schistosomiasis are largely ineffective at improving child health outcomes. ${ }^{78}$ These results are complemented by modelling studies that have shown the current recommendations for MDA are not predicted to achieve the WHO targets of morbidity control or elimination as a public health problem within the specified timelines. ${ }^{9}{ }^{10}$ This is especially pronounced in high prevalence settings, where achieving targets with MDA alone is not likely unless the intervention coverage is increased to $85 \%$ in school-aged children (SAC) and $40 \%$ of individuals over the age of 15 years. ${ }^{11}$ The practical implementation of such changes would be challenging given the current target of $75 \%$ SAC coverage remains elusive in most locations. ${ }^{1}$ The inclusion of additional interventions, such as snail (vector) management and water sanitation and hygiene (WASH) activities, has been predicted to reach the targets within the time frame specified in the NTD Road Map to $2030,{ }^{9}$ yet remain as secondary or optional components to the MDA-focused agendas of many donors, policymakers and researchers. In addition, once the targets are reached, there remain uncertainties about how feasible it will be to maintain low prevalence levels in some areas without continued rounds of PC, potentially in perpetuity. ${ }^{12}$

This perpetuation of biomedical solutions to complex infectious disease problems delivered through vertical programmes, despite evidence favouring more holistic approaches, has been well documented and critiqued. ${ }^{1314}$ These interventions have been largely devised outside of the recipient localities and are often implemented without the genuine input from relevant public authorities and intended beneficiaries. ${ }^{15}$ This is compounded by the disconnect between the development of evidence to justify and support intervention implementation and the actual evidence needs of policymakers and practitioners in endemic countries for decision-making regarding control and elimination activities. ${ }^{16}$ Together these processes undermine the domestic ownership of health issues and solutions because they position the decisionmaking power around prioritisation, service delivery and evaluation largely outside of the countries themselves. ${ }^{1718}$ And, ultimately, this runs antithetical to the principles espoused in the most recent NTD Road Map to 2030.

To shift these dynamics, it is vital to integrate more diverse perspectives, particularly from those embedded within the endemic settings. Not only will this enhance country ownership, but will also improve the design, delivery, and, ultimately, the impact of interventions. Schistosomiasis as a complex disease problem is embedded in space-specific social, economic, biological and environmental systems. The dynamic relationships between these systems determine the prevalence of the disease and the effectiveness of interventions, which are not captured in linear theories of change or reductionist methods of evaluation. Individuals who themselves are embedded in these systems can provide critical insights as to these relationships and the potential impacts of interventions over time. Mass deworming activities, in particular, are often implemented with complementary components, such as WASH or nutrition activities-all of which influence each other to produce a summative effect different from that of the individual components. ${ }^{19}$ In addition, population-based interventions generally should be viewed as dynamic, longitudinal processes, as interactions with local ecologies and social systems affect the interventions and intervention settings over time. ${ }^{2021}$ These features configure non-linear relationships, especially feedback loops and phase transitions, which are not accounted for in the prevalent deterministic, linear models of change. ${ }^{2022}$

This study aimed to develop evidence for decisionmaking in response to the needs of policymakers and practitioners from the Uganda Ministry of Health $(\mathrm{MoH})$, while incorporating the complexity of schistosomiasis transmission and control activities. This was accomplished by (1) capturing their perspectives on schistosomiasis transmission using qualitative participatory modelling, and (2) using the participatory modelling outputs to inform mathematical model simulations in response to the evidence needs. Participatory methods have been previously used to inform health policy, practice and evaluation, most notably in relation to non-communicable diseases $^{23}$ and accompanying risk factors, ${ }^{24}$ but has not been linked explicitly to modelling work and the development of evidence for decision-making in this way or context. Similar variations of this protocol used for this study have been previously described in the context of energy policy ${ }^{25}$ and ecosystem management, ${ }^{26}$ but not implemented outside of higher income countries in the context of NTDs.

\section{METHODS}

Various types of participatory modelling have been used to generate shared conceptual depictions of complex health issues, following on from the prominent work on 
poverty reduction and development led by the World Bank and other research through the late 20th century, such as Robert Chambers' work on Participatory Rural Appraisal. ${ }^{27}$ Systems mapping is a type of participatory modelling, used to elicit and quantify diverse perspectives from a variety of actors on causal relationships within complex systems. The process ends with a 'systems map', a diagram of explicit factors, causally linked to one another, which visually depicts a defined 'system' from the perspective of participating discussants ${ }^{25}$; examples of similar outputs include causal loop diagrams and stock-and-flow diagrams. More recently, systems mapping has been embraced by some working on computational modelling and simulation methods as a way to inform models with 'real-world' information vis-a-vis the outputs of 'purposeful learning processes for action that engage the implicit and explicit knowledge of stakeholders to create formalised and shared representations of reality'. ${ }^{28}$ Depending on the aims and context, systems maps can be used to guide, inform, and even employed as the frameworks for simulations and other modelling activities. In health, systems maps have previously been used in calls for applying a systems epidemiology framework to schistosomiasis. ${ }^{29}$ In this study, systems maps produced using the Participatory Systems Mapping (PSM) method with individuals working on schistosomiasis in Uganda framed the form and content of subsequent modelling activities and explicitly informed model parameter specifications.

\section{Participants}

Individuals working on schistosomiasis control and elimination activities from the national, district and village levels within the Ugandan health system were invited to participate in workshops in October of 2019. The aim was to implement a process which captured their perspectives and then incorporate these into the development of evidence for decision-making which was directly responsive to their needs. Thirty-three individuals from the national, district and village levels participated in two PSM workshops over 3 days. The participants were purposively invited to the workshop in consultation and coordination with the Uganda MoH Vector Control Division and the Uganda-UK Health Alliance. Individuals were selected from low, moderate and high transmission settings, with the aim of capturing diverse perspectives and encouraging discussions across transmission settings. Those individuals from the national level were from $\mathrm{MoH}$ departmental headquarters and two non-governmental organisations involved in schistosomiasis control activities. Individuals from the district level included district vector control officers and district health officers within the $\mathrm{MoH}$ organisational structure. Individuals from the village level were members of village health teams (VHTs), volunteer community health workers also organised within the $\mathrm{MoH}$. One workshop with national-level and district-level representatives took place in Kampala, and the second workshop with members of VHTs took place in Jinja. Participants were reimbursed or provided with transportation, accommodation and sustenance to facilitate their participation.

\section{PSM workshops}

The workshops included presentations on the fundamentals of modelling and evidence related to schistosomiasis, as well as small and large group discussions on schistosomiasis control and elimination strategies and evaluation of the PSM outputs. In addition, participants were provided background on how systems maps could be used to define the parameters of simulation activities. The PSM exercises followed the process described by Barbrook-Johnson and Penn from the Centre for Evaluating Complexity Across the Nexus (CECAN). ${ }^{25}$ After being provided with background and instructions on PSM, small groups of four to eight participants were formed based on health system level, to ease potential pressures of speaking up in the presence of workplace superiors.

The PSM was managed in each group by one or two facilitators and a note-taker. To begin the exercise, each group was given the prompt 'schistosomiasis transmission' and instructed to individually brainstorm factors which directly or indirectly impact transmission. These were then brought together and linked causally through group debate and consensus over course of a day. The systems maps were initially built using erasable paper, markers and sticky notes. Digitised versions of the systems maps were presented to the participants for validation, and the digital versions were corrected accordingly.

Subsequent large group discussions used the systems maps as tools to describe the impact of specific factors represented in the systems maps, and describe how interventions might be designed and implemented to influence key points in the system to drive down schistosomiasis transmission. The maps were also used as a point of departure to evaluate the potential causes for the lack of effectiveness of current interventions, in particular MDA, on producing long-term, sustained reductions in schistosomiasis transmission.

\section{Generation of the aggregated systems map}

To incorporate the group maps into a full systems map, the factors from each group were combined and standardised to a minimal extent (eg, 'children playing/swimming' and 'children playing in water' were standardised to 'playing/swimming') for a master list of factors described by the participants as directly or indirectly related to schistosomiasis transmission (see online supplemental materials 1). Each group map was then reimplemented with the standardised factor names as two-dimensional adjacency matrices, with cell values $\left(\alpha_{i, j}\right)$ equal to +1 (positive relationship), -1 (negative relationship) or 0 (no relationship) from the row factor $(i)$ to the column factor $(j)$. Factors from the master list not included in a given group map were added to the corresponding adjacency matrix (with $\alpha_{i, j}=0$ ) as needed for a conformable set of matrices. Matrix addition was used to combine the 
four matrices, with the resultant $\alpha_{i, j}$ values (ranging from -4 to +4$)$ representative of the generalised importance of each factor, captured by frequency with which each relationship was mentioned across the systems maps.

The full adjacency matrix was exported as a weighted edge list and projected as the full systems map. Structural analysis of the full map considered network centrality measures to identify the factors of greatest interest. ${ }^{30}$ This method of network analysis was chosen a priori as part of the study protocol to reduce researcher bias in the identification of factors of interest, and also with the intention to use the same method in subsequent research comparing systems maps over time and between countries. These were considered along with the outcomes of the small and large group discussions to define the purpose of the subsequent model simulations and inform the model parameter specifications. Following the workshops, the participants provided feedback on the summaries of the activities and discussions to ensure that the notes accurately reflected the content, and verified the final digitised versions of the systems maps. Additional inputs from participants were included through individual discussions as the modelling process progressed, with respect to their time commitments through 2020.

\section{Model and simulation overview}

In terms of appropriate methods for incorporating complexity, individual-based modelling is able to accommodate the stochastic, non-linear, and dynamic interactions between humans and the environment in the transmission cycle of schistosomiasis. In addition, and of importance for the purposes of this study, it is scalable (from a village to a national setting), adaptable across contexts, flexible in the types of information that can be included and inclusive in the ways that outputs can be communicated. The SCHISTOX model employed in this study is an individual-based simulation model developed by Graham $e t a l .^{31}$ In addition to the suitability of its stochastic framework and inclusion of both Schistosoma mansoni and S. haematobium species, the SCHISTOX model has the distinct advantage in its development as an open-source repository on GitHub, ${ }^{32}$ which can be run in Julia or through an $\mathrm{R}$ wrapper. This latter point is particularly important in that it allows for more straightforward communication with workshop participants, as they are able to see and work with the actual coding, when compared with other stochastic models of schistosomiasis transmission, which were reported as being seen by participants as 'black boxes'. Model parameters, categorised as human population, parasite population, transmission or control, can be explicitly specified based on a given context and available information. Given the prevalence of both $S$. haematobium and S. mansoni across Uganda, models were run to include the relevant parameter specifications adapted for both species. Parameters were defined by Graham et al's SCHISTOX publication, ${ }^{31}$ the SCHISTOX model documentation on GitHub (last accessed October 2021), ${ }^{32}$ personal correspondence with the model developers and in consultation with workshop participants. As discussed next, the primary focus of the simulations presented here was to observe how changes in the population water contact parameter would impact the prevalence, with specific reference to the WHO target timelines, while holding the other initialisation parameter values constant. All parameter specifications used in this study can be found in online supplemental materials 2.

To initialise the simulations, each species-specific model was run for 100 years under high prevalence scenarios to establish epidemiological equilibrium within the population. The aim of the simulations followed the results of the PSM workshops and subsequent discussions with participants. In particular, participants were interested in the potential impacts of limiting water contact and had suggested a variety of context-specific and place-specific interventions. Some of these interventions included providing gum boots to rice farmers and fisher-folk, clothes washing stations and bathing shelters (see online supplemental materials 4 , theme 2 for additional details from participants-related water contact). Given the aim to provide generalised guidance to these diverse situations, exploratory age-specific water contact was simulated over a series of proportional reductions which could then be applied in local contexts and decision-makers to scale preferred interventions.

To provide guidance on the potential impact of intervention combinations, four scenarios were considered in high prevalence $S$. mansoni and S. haematobium settings. Reduction in water contact was considered alongside MDA implementation. MDA coverage for SAC was simulated at two levels in accordance with the current WHO and national guidelines: (1) the most recent reported median coverage for high prevalence districts $(46 \%$, range), ${ }^{33}$ and (2) the recommended target coverage of $75 \%$. The reported median coverage for high prevalence districts in Uganda was selected to provide a relevant reference point, relative to the recommended target coverage, for district and subdistrict decision-makers. The simulations were run for 10 years in the SCHISTOX $\mathrm{R}$ interface.

\section{Patient and public involvement}

There were no funds or time available for patient or public involvement in this study. Additional research based on this study involving individuals with schistosomiasis is being developed. However, we are cognisant of the effects of the ongoing COVID-19 pandemic in the areas where we work and are putting our efforts into the pressing needs of the health workers and patients ahead of a research agenda.

\section{RESULTS}

The systems maps produced through PSM presented complex, dynamic perspectives on the transmission of schistosomiasis across Uganda. The participants 


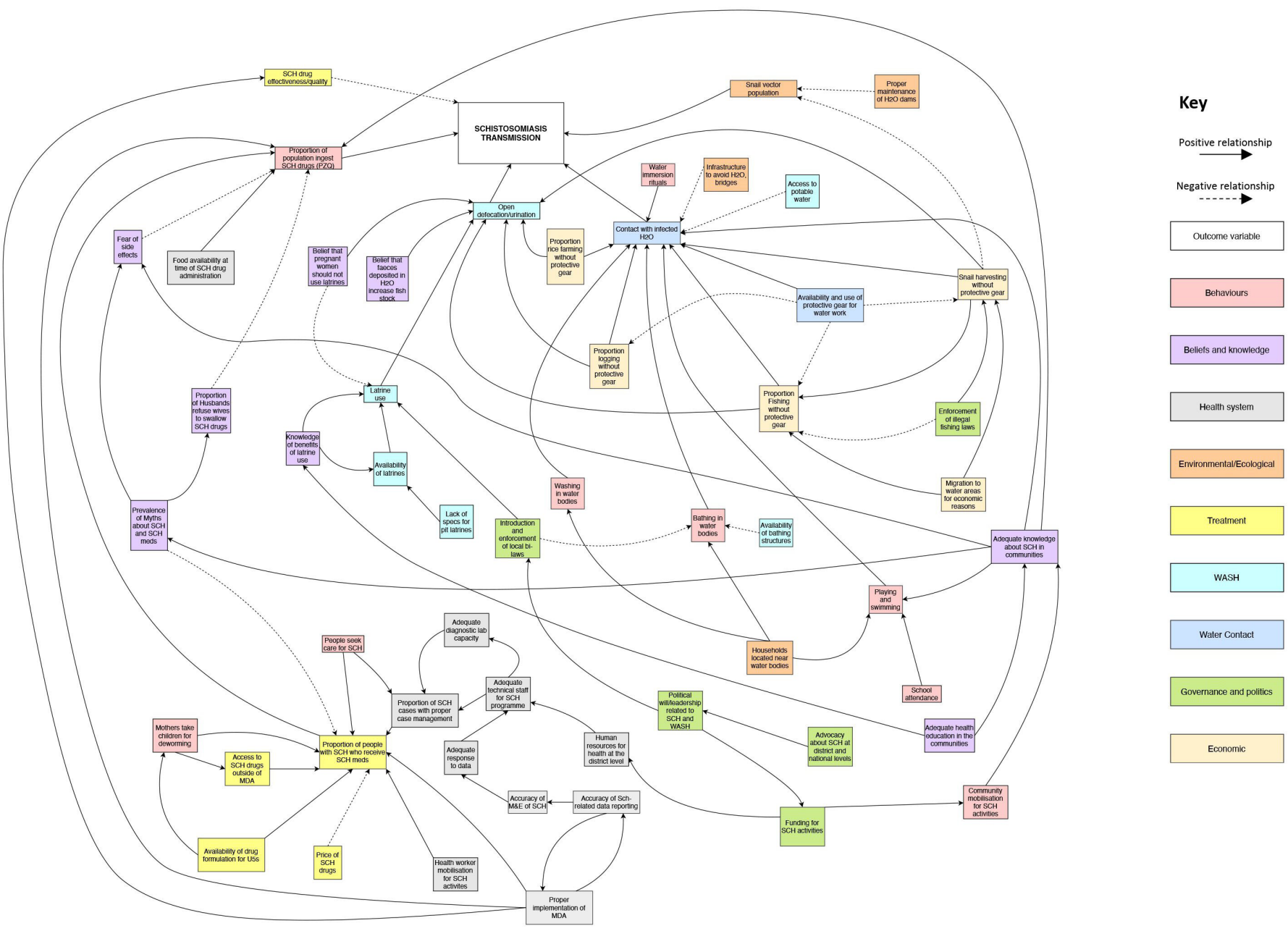

Figure 1 Aggregate systems map. M\&E, monitoring and evaluation activities; MDA, mass drug administration; PZQ, praziquantel; SCH, schistosomiasis; U5s, children aged less than five years; WASH, Water Sanitation and Hygiene activities.

identified key points of potential intervention as centred around water contact from economic, household, hygiene and leisure activities. The aggregate systems map (figure 1) shows five factors directly influencing schistosomiasis transmission: open defecation/urination, ingestion of the schistosomiasis drug praziquantel, quality/ standard of schistosomiasis drugs (drug efficacy), population of snail vectors and contact with infested water. The factors identified as directly or indirectly effecting schistosomiasis transmission were across eight categories: individual behaviours, beliefs and knowledge, health system components and activities, environmental/ecological, schistosomiasis treatment/drugs, WASH, water contact activities and governance/politics. The small group maps can be found in online supplemental materials 3 . The key discussion points which emerged from the small and large group discussions are summarised in table 1 and online supplemental materials 4 .

To further analyse the structure of the aggregate systems map, network centrality metrics were determined for each of the factors. In line with the small and large group discussions, the factors with the highest degree centrality, that is, those with the highest number of incident links from and to other factors, were 'contact with infested water' and 'open defecation and urination' (see online supplemental materials 5 for all network centrality metrics). This suggests that these are vital intervention points in the system of schistosomiasis transmission from the perspectives of the workshop participants.

As discussed above, following on from the outputs of the participatory modelling and analysis of the aggregate map, the simulation activities centred around reductions in water contact. As an exploratory step, simulated reductions in the age-specific water contact in relation to the initialisation parameters were tested. Results showed that decreasing contact with infected water by $75 \%$ across all ages in high $S$. mansoni prevalence settings, while holding all else equal, achieved morbidity control within 20 years and achieved elimination as a public health problem within 30 years. In high $S$. haematobium settings, water contact reductions of $75 \%$ achieved morbidity control within 15 years and elimination within 20 years (all of the water contact exploratory simulation results can be found in online supplemental materials 6).

Further simulations were used to compare the impact of water contact reductions with current and target MDA coverage levels on the prevalence of schistosomiasis, individually and in combination (figure 2). The simulation 
Table 1 Key discussion points and examples from Participatory Systems Mapping workshop and follow-up

\begin{tabular}{lll} 
No $\quad$ Key discussion point & Specific examples \\
\hline 1 & $\begin{array}{l}\text { Water contact is an especially important transmission/ } \\
\text { potential control point which allows for substantial } \\
\text { flexibility in the design of interventions and control of } \\
\text { implementation components at the local level. }\end{array}$ & $\begin{array}{l}\text { Specific control points were economic activities (fishing, rice } \\
\text { farming and snail harvesting), household activities (washing, } \\
\text { fetching water) and hygiene activities (bathing, latrine use). }\end{array}$ \\
$\begin{array}{l}\text { The only group level focused on MDA implementation } \\
\text { was comprised of members of village health teams } \\
\text { (VHTs); the district-level and national-level groups } \\
\text { mentioned the intervention, but not in detail. }\end{array}$ & $\begin{array}{l}\text { As the group directly responsible for MDA implementation, } \\
\text { the VHTs detailed material support (bags to carry medicines, } \\
\text { fuel, salaries) as factors influencing MDA implementation. }\end{array}$ \\
$\begin{array}{l}\text { Individuals from all groups discussed the lack of } \\
\text { available treatment in communities outside of MDA } \\
\text { implementation periods. }\end{array}$ & $\begin{array}{l}\text { The lack of treatment availability in health facilities leads to } \\
\text { the inability to provide proper case management with the } \\
\text { Communication related to schistosomiasis transmission } \\
\text { and interventions needs to be improved between the } \\
\text { national, district and village levels. }\end{array}$ & $\begin{array}{l}\text { There was a disconnect in the dissemination of updated, } \\
\text { relevant and useful materials from the national to the } \\
\text { subnational levels, specifically these concerns were the need } \\
\text { for translation into local languages and the provision of hard } \\
\text { copy formats. }\end{array}$ \\
$\begin{array}{ll}\text { The system for collecting data related to schistosomiasis } \\
\text { is inefficient and ineffective for routine use and } \\
\text { facilitating responses. }\end{array}$ & $\begin{array}{l}\text { Data collection and feedback are a patchwork of reliability } \\
\text { and completely dependent on the individual data collector at } \\
\text { the community level and the aggregator at the district level. }\end{array}$
\end{tabular}

MDA, mass drug administration; MDA, mass drug administration (preventative chemotherapy).

results showed that employing MDA for SAC as the sole intervention, at both the current median coverage level in high prevalence districts in Uganda $(46 \%)$, and the

A Heavy burden prevalence $S$. mansoni setting

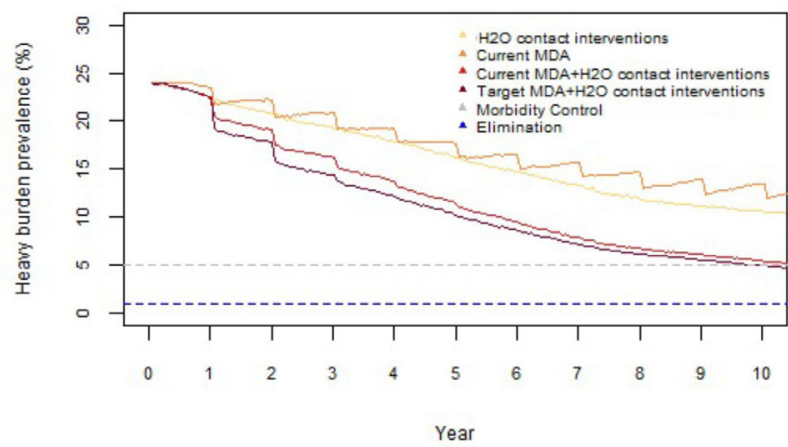

C Population prevalence S. mansoni setting

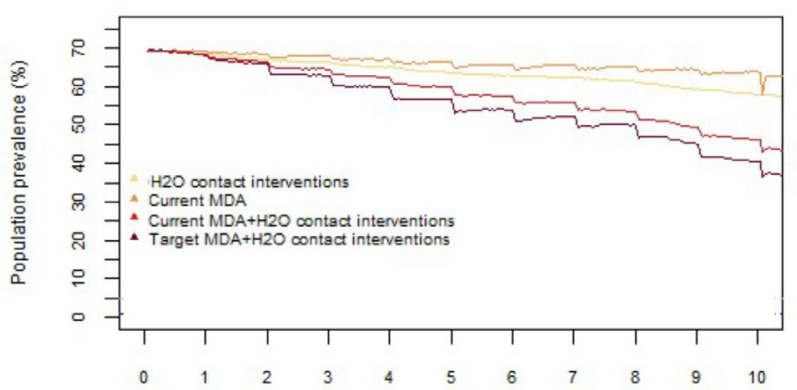

Year policy-recommended target of $75 \%$ coverage, did not achieve either morbidity control or elimination within the 10 -year time period. The results showed that combining

B Heavy burden prevalence S. haematobium setting

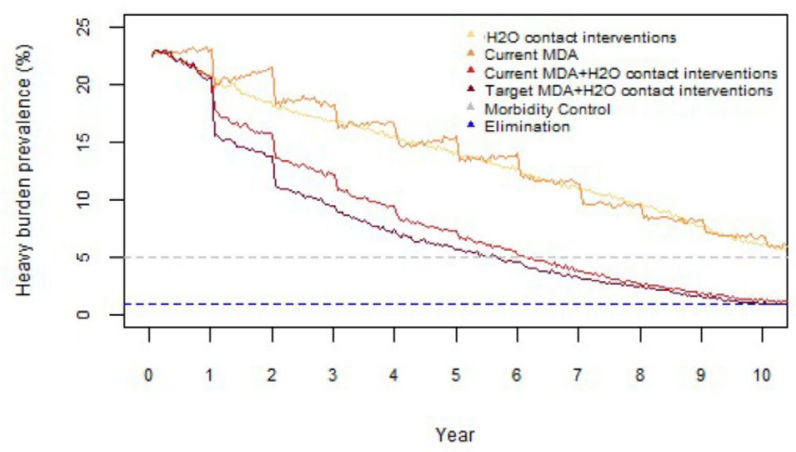

D Population prevalence $S$. haematobium setting

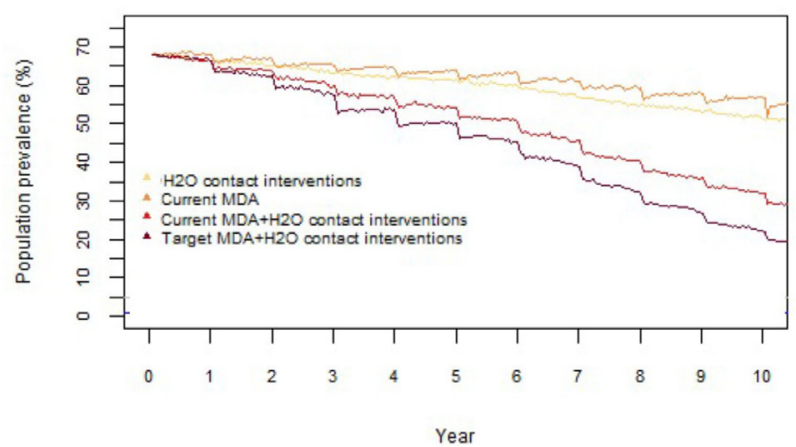

Figure 2 A-D. MDA and water contact reduction simulation results. Note: $\mathrm{H}_{2} \mathrm{O}$ contact interventions, implementation of water contact reduction interventions to decrease contact by $75 \%$; current MDA, most recent reported median coverage of schoolaged children (SAC) for high prevalence districts in Uganda (46\%); target MDA, recommended target coverage of SAC in high prevalence districts of $75 \%$; these results were not adjusted for diagnostic sensitivity. MDA, mass drug administration. 
water contact reduction interventions at $75 \%$ with the current $(46 \%)$ or target (75\%) MDA coverage reached the most recent WHO's NTD Road Map to 2030 targets of morbidity control and elimination in both $S$. mansoni and $S$. haematobium settings within 10 years. While the simulations indicated that morbidity control and elimination could be achieved, as these indicators pertain to high intensity infections, it should be noted that population prevalence did not achieve $5 \%$ or $1 \%$ targets in settings with either species within 10 years (figure 2).

\section{DISCUSSION}

This study used PSM to elicit and depict the perspectives of Ugandan policymakers and practitioners on factors related to schistosomiasis transmission and interventions. Focus group discussions and follow-up interviews provided additional information and insights as to their evidence needs and guided the subsequent modelling activities. These outputs framed individual-based modelling simulations and were incorporated into the model parameter specifications. Simulations were used to predict the impacts of water contact reductions in communities of high S. haematobium and S. mansoni prevalence settings. The combination of PSM and individualbased modelling was a rich strategy which explicitly integrated the perspectives of national and subnational policymakers and practitioners into the development of evidence for decision-making related to schistosomiasis control and elimination activities.

The visualisations of the schistosomiasis transmission system were produced by national-level, district-level, and village-level policymakers and practitioners involved in schistosomiasis control and elimination activities. The systems maps indicated causal effects and the directionality of these effects (positive or negative) by linking factors where relationships were perceived to exist. The digitised versions of the maps served as depersonalised expressions of consensus by small groups that facilitated conversations in the large group about difficult topics, related in particular to resources, data and the lack of sustained reductions in prevalence after years of deworming interventions. These discussions may not have otherwise taken place openly given the social dynamics between district-level and national-level participants. Feedback from participants indicated that they hoped to use the systems maps to advocate for resources they deemed necessary from within the Uganda and among international donors and aid agencies, as the maps were viewed as leverage in a system of top-down decisionmaking around schistosomiasis control activities. PSM encouraged critical thinking and provided the space to develop potential solutions based on lived and professional experiences. From the systems maps, it was clear that participants at the national, district and village levels were most focused on factors that increase or decrease infested water contact, a key intervention point in the schistosomiasis transmission cycle. This perspective was used to guide the model simulation activities.

The SCHISTOX individual-based model by Graham $e t$ $a \ell^{\beta 1}$ was employed to simulate the impacts of age-specific reductions in water contact under various scenarios. The results showed that employing MDA alone, at either the current or target levels of SAC coverage, did not result in achieving the most recent NTD Road Map to 2030 targets of morbidity control or elimination in high prevalence settings within 10 years. However, when combined with water contact reduction activities, morbidity control was reached in $S$. mansoni settings and morbidity control and elimination were achieved in S. haematobium settings within the same 10-year time period. These outcomes were modelled in the context of high prevalence levels and may not be generalisable to low or moderate prevalence settings.

There were several important insights from this study relevant to the broader context of the NTD Road Map to 2030 and the global strategies for achieving schistosomiasis morbidity control and elimination. The PSM supported critiques about mass deworming strategies as vertical, top-down interventions. ${ }^{34}$ The simulations provided further evidence that MDA alone will not achieve the prevalence reduction targets. ${ }^{9}{ }^{10}$ Individuals from VHTs were the only group to specifically discuss the implementation of MDA in relation to schistosomiasis transmission, most likely because they were directly responsible for carrying out these activities. The lack of access to treatment for routine care within the communities, leading to the inability to provide adequate case management, was an important gap highlighted in the context discussions about MDA.

Interventions to reduce exposure to infected water were not included in the WHO's NTD Road Map to 2030, despite being the reported driver to elimination of schistosomiasis in previous case studies in the same document. In this study, water contact was described as the key potential intervention point in the schistosomiasis transmission cycle by participants and reduction in water contact was shown in simulations to be an important component leading to decreased prevalence and eventual elimination. Previous empirical work by Knopp et al showed that behavioural and educational interventions were not as effective at reducing schistosomiasis prevalence as MDA alone or as integrated components, ${ }^{34}$ although, as has been discussed elsewhere, ${ }^{4}$ the integrated components may not have been implemented widely enough to generalise the findings.

There is clearly still a need to better understand the feasibility and costs of water contact reduction interventions. In most places with a high prevalence of schistosomiasis infections, contact with local water bodies underpins the social, economic and hygiene activities of daily life. Therefore, any adaptations to these activities would need to be developed and led from within communities in order to achieve meaningful reductions in water contact. The degree to which this is feasible, and the extent of 
the impact, is entirely context specific and dependent on holistic approaches to funding and implementation. In addition, a bigger push toward community-wide MDA in all relevant high-prevalence areas, improvements in diagnostics, the prospects of a vaccine and a host of other innovative technologies will undoubtedly play a role in moving toward the elimination of schistosomiasis as a public health problem.

It is also important to acknowledge the challenges with implementing participatory modelling and the potential issues with its outputs. Clearly the biggest limitation of the work presented here is that participatory modelling activities are very resource intensive, both in time and money. Prior to the implementation of the activities, it requires relationship building to foster credibility and buy-in from participants. In terms of outputs, fundamentally, the systems maps are abstractions of reality. These are negotiated representations of individual perspectives which do not 'objectively' nor entirely capture the system which results in schistosomiasis transmission. In this way, actually, the outputs of participatory modelling are akin to the outputs of mathematical modelling: both are inherently biased by the composition of individuals whose inputs drive and shape these processes. One of the broader aims of this work was to explore how we might explicitly use these biases to allow for locally fostered approaches to evidence for decision-making.

\section{CONCLUSION}

The WHO's NTD Road Map to 2030 calls for a country-led process supported by partners. If this is meant beyond rhetoric, partner organisations need to engage with policymakers and practitioners in endemic countries, not only as the recipients of evidence for decisionmaking or facilitators of interventions produced outside the communities, but as individuals capable of driving these processes. This study demonstrates one of many possible strategies to integrate a wider range of perspectives in the form of individuals directly involved in the policy, oversight, and implementation of schistosomiasis control and elimination strategies within endemic countries. Inclusivity and the flexibility to allow innovation to be driven by a more diverse set of voices and experiences will push the sustainable reduction in the burden of schistosomiasis by 2030 .

\author{
Author affiliations \\ ${ }^{1}$ Firoz Lalji Institute for Africa, LSE, London, UK \\ ${ }^{2}$ Department of International Development, LSE, London, UK \\ ${ }^{3}$ Vector Control Division, Republic of Uganda Ministry of Health, Mayuge, Uganda \\ ${ }^{4}$ Vector Control Division, Republic of Uganda Ministry of Health, Pakwach, Uganda \\ ${ }^{5}$ Department of Global Health and Development, London School of Hygiene and \\ Tropical Medicine, London, UK \\ ${ }^{6}$ Uganda-UK Health Alliance, Kampala, Uganda \\ Twitter Melissa Parker @0rcid 0000-0003-0829-2741 and Tim Allen @0rcid \\ 0000-0003-1866-0181
}

Acknowledgements We would like to acknowledge Dr Moses Adriko (Uganda Ministry of Health Vector Control Division) and Dr Georgina Pearson (London School of Economics and Political Science; Queen Margaret University, Edinburgh) for their assistance in conducting the participatory workshops. We would like to thank $\mathrm{Dr}$ Elizabeth Storer (London School of Economics and Political Science) for her input and thoughtful insights through the writing process. We would also like to thank Dr Tiziana Leone and the participants of LSE's Global Health Initiative Reading Group for comments on earlier iterations of this manuscript. We thank the health workers, practitioners and policymakers working in Uganda who participated in the workshops, provided feedback throughout the processes described in this paper and continue to work with us while balancing their responsibilities through the COVID-19 pandemic. Finally, we thank Dr Matthew Graham and Dr Diepreye Ayabina from University of Oxford's Big Data Institute for their invaluable assistance and insights on working through their SCHISTOX model. Their willingness to provide support to the end of creating a truly open-source individual-based model reflects the best we can hope for in the future of scientific research.

Contributors CF, MP, SK, BO, NO and TA implemented the study and provided interpretations of the data. CF guided the conceptualisation and investigation, wrote the first draft of the article, and guided the writing, review, and editing. All authors contributed to drafting the work or revising it critically for important intellectual content, and all authors have read and approved the final version. All authors agree to be accountable for all aspects of the work in ensuring that questions related to the accuracy or integrity of any part of the work are appropriately investigated and resolved. CF accepts full responsibility as guarantor of the overall content, including the finished work, the conduct of the study, access to the data, and control over the decision to publish.

Funding This study was funded by Research England's Bloomsbury Science, Engineering, and Technology (SET) grant programme (Localised Evidence and Decision-making Project Reference BSA04; Award Reference CCF17-7779).

Disclaimer The funder of the study had no role in the study design, data collection, data analysis, data interpretation or writing of the report. The authors had full access to all the data in the study and accept responsibility for the decision to submit for publication.

Competing interests None declared.

Patient consent for publication Not required.

Ethics approval This study involves human participants and ethical clearance and permission for the relevant components of this study were granted from the London School of Economics and Political Science Research Ethics Committee (reference number 000914) and the Uganda National Council for Science and Technology (research registration number HS1285ES). Written informed consent was obtained from all workshop participants.

Provenance and peer review Not commissioned; externally peer reviewed.

Data availability statement All data relevant to the study are included in the article, uploaded as supplemental information, or available upon reasonable request.

Supplemental material This content has been supplied by the author(s). It has not been vetted by BMJ Publishing Group Limited (BMJ) and may not have been peer-reviewed. Any opinions or recommendations discussed are solely those of the author(s) and are not endorsed by BMJ. BMJ disclaims all liability and responsibility arising from any reliance placed on the content. Where the content includes any translated material, BMJ does not warrant the accuracy and reliability of the translations (including but not limited to local regulations, clinical guidelines, terminology, drug names and drug dosages), and is not responsible for any error and/or omissions arising from translation and adaptation or otherwise.

Open access This is an open access article distributed in accordance with the Creative Commons Attribution Non Commercial (CC BY-NC 4.0) license, which permits others to distribute, remix, adapt, build upon this work non-commercially, and license their derivative works on different terms, provided the original work is properly cited, appropriate credit is given, any changes made indicated, and the use is non-commercial. See: http://creativecommons.org/licenses/by-nc/4.0/.

\section{ORCID iD}

Cristin Alexis Fergus http://orcid.org/0000-0002-7819-2087

\section{REFERENCES}

1 World Health Organization. Ending the neglect to attain the sustainable development goals: a road map for neglected tropical diseases 2021-2030, 2020. Available: https://apps.who.int/iris/ handle/10665/338565 [Accessed 9 Apr 2021]. 
2 Malecela MN, Ducker C. A road map for neglected tropical diseases 2021-2030. Trans $R$ Soc Trop Med Hyg 2021;115:121-3.

3 Tchuem Tchuenté L-A, Rollinson D, Stothard JR, et al. Moving from control to elimination of schistosomiasis in sub-Saharan Africa: time to change and adapt strategies. Infect Dis Poverty 2017;6:42.

4 Mazigo HD. Participatory integrated control strategies and elimination of schistosomiasis in sub-Saharan Africa. Lancet Glob Health 2019;7:e998-9.

5 Evan Secor W. Water-based interventions for schistosomiasis control. Pathog Glob Health 2014;108:246-54.

6 Parker M, Allen T. De-politicizing parasites: reflections on attempts to control the control of neglected tropical diseases. Med Anthropol 2014;33:223-39.

7 Taylor-Robinson DC, Maayan N, Donegan S, et al. Public health deworming programmes for soil-transmitted helminths in children living in endemic areas. Cochrane Database Syst Rev 2019;9:CD000371.

8 Welch VA, Ghogomu E, Hossain A, et al. Mass deworming to improve developmental health and wellbeing of children in lowincome and middle-income countries: a systematic review and network meta-analysis. Lancet Glob Health 2017;5:e40-50.

9 Li EY, Gurarie D, Lo NC, et al. Improving public health control of schistosomiasis with a modified who strategy: a model-based comparison study. Lancet Glob Health 2019;7:e1414-22.

10 Toor J, Rollinson D, Turner HC, et al. Achieving elimination as a public health problem for Schistosoma mansoni and S. haematobium: when is community-wide treatment required? $\mathrm{J}$ Infect Dis 2020;221:S525-30.

11 Toor J, Alsallaq R, Truscott JE, et al. Are we on our way to achieving the 2020 goals for schistosomiasis morbidity control using current World Health Organization guidelines? Clin Infect Dis 2018;66:S245-52.

12 Ayabina D, Kura K, Toor J, et al. Maintaining low prevalence of Schistosoma mansoni: modeling the effect of less frequent treatment. Clin Infect Dis 2021;72:S140-5.

13 Atun RA, Bennett S, Duran A, Europe WHORO for, Policies EO on HS and, Network (HEN) HE. When do vertical (stand alone) programmes have a place in health systems? 2008. Available: https://apps.who.int/iris/handle/10665/107977 [Accessed 23 Mar 2020].

14 World Health Organization Maximizing Positive Synergies Collaborative Group, Samb B, Evans T, et al. An assessment of interactions between global health initiatives and country health systems. Lancet 2009;373:2137-69.

15 Packard RM. A history of global health: interventions into the lives of other peoples, 2016.

16 Burchett HED, Mayhew SH, Lavis JN, et al. The usefulness of different types of health research: perspectives from a low-income country. evid policy 2015;11:19-33.

17 Gill S, Benatar S. Global health governance and global power: a critical commentary on the Lancet-University of Oslo Commission report. Int J Health Serv 2016;46:346-65.
18 Bruen C, Brugha R. A ghost in the machine? Politics in global health policy. Int J Health Policy Manag 2014;3:1-4.

19 Nikolay B, Mwandawiro CS, Kihara JH, et al. Understanding heterogeneity in the impact of national neglected tropical disease control programmes: evidence from school-based Deworming in Kenya. PLoS Negl Trop Dis 2015;9:e0004108.

20 Galea S, Riddle M, Kaplan GA. Causal thinking and complex system approaches in epidemiology. Int J Epidemiol 2010;39:97-106.

21 Petticrew M. Time to rethink the systematic review catechism? Moving from 'what works' to 'what happens'. Syst Rev 2015;4:36.

22 Petticrew M, Knai C, Thomas J, et al. Implications of a complexity perspective for systematic reviews and Guideline development in health decision making. BMJ Glob Health 2019;4:e000899.

23 Finegood DT, Merth TDN, Rutter H. Implications of the Foresight obesity system map for solutions to childhood obesity. Obesity 2010;18:S13-16.

24 Friel S, Pescud M, Malbon E, et al. Using systems science to understand the determinants of inequities in healthy eating. PLoS One 2017;12:e0188872.

25 Barbrook-Johnson P, Penn A. Participatory systems mapping for complex energy policy evaluation. Evaluation 2021;27:57-79.

26 Mehryar S, Sliuzas R, Schwarz N, et al. From individual fuzzy cognitive maps to agent based models: modeling multi-factorial and multi-stakeholder decision-making for water scarcity. J Environ Manage 2019;250:109482.

27 Chambers R. Participatory mapping and geographic information systems: whose MAP? Who is Empowered and who Disempowered? who gains and who loses? Electronic J Inform Syst Develop Countries 2006;25:1-11.

28 Voinov A, Jenni K, Gray S, et al. Tools and methods in participatory modeling: selecting the right tool for the job. Environmental Modelling \& Software 2018;109:232-55.

29 Krauth S, Balen J, Gobert G, et al. A call for systems epidemiology to tackle the complexity of schistosomiasis, its control, and its elimination. Trop Med Infect Dis 2019;4:21. doi:10.3390/ tropicalmed4010021

30 Papageorgiou K, Singh PK, Papageorgiou El, et al. Participatory modelling for poverty alleviation using fuzzy cognitive maps and OWA learning aggregation. PLoS One 2020;15:e0233984.

31 Graham M, Ayabina D, Lucas TC, et al. SCHISTOX: an individual based model for the epidemiology and control of schistosomiasis. Infect Dis Model 2021:6:438-47.

32 Graham M. Schistoxpkg.jl, 2021. Available: https://github.com/ mattg3004/Schistoxpkg.jl

33 ESPEN. Uganda|schistosomiasis, expanded special project for elimination of neglected tropical diseases (ESPEN). Available: https://espen.afro.who.int/countries/uganda [Accessed 5 Apr 2021].

34 Knopp S, Person B, Ame SM, et al. Evaluation of integrated interventions layered on mass drug administration for urogenita schistosomiasis elimination: a cluster-randomised trial. Lancet Glob Health 2019;7:e1118-29. 